\title{
D-06
}

\section{LA ACTIVIDAD DE LOS MERCADOS FORMALES DE AGUA EN LA CUENCA DEL SEGURA}

\author{
Calatrava, J (1) (P), Martínez-Granados, D (2)
}

\author{
${ }^{1}$ Profesor Titular de Universidad, E.T.S. de Ingeniería Agronómica, Universidad Politécnica \\ de Cartagena, Paseo Alfonso XIII 48, 30203 Cartagena, j.calatrava@upct.es \\ 2 Investigador, E.T.S. de Ingeniería Agronómica, Universidad Politécnica de Cartagena, \\ Paseo Alfonso XIII 48, 30203 Cartagena, david.martinez@upct.es
}

RESUMEN: La cuenca del Segura, en el sureste de España, es una de las zonas con mayor nivel de escasez de agua, no solo de España, sino de toda Europa. Entre las múltiples medidas tomadas por la Administración española para hacer frente a la escasez de agua en esta y otras cuencas del país, en 1999 se reformó la Ley de Aguas para introducir los mercados de agua en el ordenamiento jurídico español y regular su funcionamiento. El objetivo que se perseguía era flexibilizar el régimen concesional del agua en España para mejorar la reasignación de los recursos hídricos, incrementar la eficiencia económica en el uso del agua y reducir el impacto económico de la escasez. Las características de la economía del agua en la cuenca del Segura suponen unas muy favorables condiciones de inicio para la actividad de mercado, por lo que el potencial para su funcionamiento es notable. Sin embargo, y pese a concentrar la mayor actividad de mercados de agua de la España continental, el volumen de intercambios de agua en esta cuenca ha sido relativamente escaso. En este trabajo se describen las escasas experiencias de mercados formales de agua en la cuenca del Segura, tanto los realizados entre usuarios de la propia Demarcación, como los celebrados con usuarios de otras cuencas y las Ofertas Públicas de Adquisición de Derechos realizadas por la Administración.

\section{Introducción}

La cuenca del Segura es una de las áreas con mayor nivel de escasez de agua de Europa. En un contexto de creciente escasez estructural, el reparto del agua genera importantes conflictos entre usuarios que se agravan durante los periódicos episodios de sequía. Entre las múltiples medidas tomadas por el Gobierno de España para hacer frente a la escasez de agua en ésta y otras cuencas, en 1999 se reformó la Ley de Aguas (Ley 46/1999) para introducir los mercados de agua en el ordenamiento jurídico español y regular su funcionamiento. Se perseguía con ello flexibilizar el régimen concesional del agua para mejorar la reasignación de los recursos hídricos, incrementar la eficiencia económica en el uso del agua y reducir el impacto económico de la escasez (Rey et al., 2014).

La Ley 46/1999 permitió el intercambio voluntario de agua entre concesionarios que lleguen a un acuerdo privado para la cesión temporal de sus derechos de uso o propiedad del agua a cambio de una compensación, previa solicitud y autorización por parte del Organismo de Cuenca correspondiente. Estos contratos de cesión temporal de derechos pueden celebrarse entre usuarios situados dentro de una misma demarcación hidrográfica, y siempre hacia usos de igual o mayor orden de prelación. Aunque este mecanismo está pensado para asignar los recursos en períodos de sequía, su funcionamiento no está restringido a éstos. Posteriormente, durante la sequía de 2005-2008 el Ministerio de Medio Ambiente permitió, mediante el RDL 15/2005 y sus prórrogas anuales, los contratos de cesión entre usuarios de diferentes cuencas como medida excepcional para aliviar los 
problemas de disponibilidad de agua de las zonas más afectadas (Garrido et al., 2013b). En el caso del Segura, los volúmenes adquiridos a usuarios del Tajo durante 2006 superaron con creces a todos los contratos de cesión celebrados en la cuenca hasta ese año.

Además de la figura de los contratos de cesión entre usuarios, la Ley 46/1999 contemplaba la posibilidad de que los Organismos de Cuenca pudiesen crear los denominados Centros de Intercambio de Derechos (CID) de uso del agua, a través de los cuales realizar ofertas públicas de adquisición de derechos (OPAD) a los titulares de concesiones interesados en cederlas temporal o permanentemente para proceder posteriormente a su cesión a otros titulares (Calatrava y Gómez-Ramos, 2009). Los primeros CID se crearon en las cuencas hidrográficas del Guadiana, Júcar y Segura en 2004, aunque no funcionaron hasta que, con el inicio de la sequía de 2005-08, el RDL 9/2006 reforzó su eficacia para que pudiesen dar respuesta también a otras demandas (Garrido et al., 2013b). Por ejemplo, además de ceder recursos a otros usuarios (objetivo inicial), los CID podrían también destinar los derechos adquiridos a usos ambientales.

Las características de la economía del agua en la cuenca del Segura (escasez crónica, acentuada variabilidad en la oferta de agua, frecuentes episodios de sequía, distribución desigual de los recursos en favor del regadío tradicional, notables diferencias en el valor del agua y existencia de infraestructuras de transporte de agua entre las principales zonas de riego) constituyen unas favorables condiciones de inicio para la actividad de los mercados de agua, por lo que el potencial para su funcionamiento es notable. Sin embargo, el volumen de intercambios de agua en esta cuenca ha sido relativamente escaso. Pese a ello, concentra la mayoría de los contratos de cesión intra-cuenca de la España continental y más de la mitad de los celebrados entre usuarios de diferentes cuencas.

En este sentido, el objetivo de este trabajo es revisar las experiencias de mercados formales de agua en la cuenca del Segura desde 1999, tanto los realizados entre usuarios de la propia Demarcación, como los celebrados con usuarios de otras cuencas y las Ofertas Públicas de Adquisición de Derechos realizadas por la Administración. Para ello se han recopilado y clasificado las transacciones de las que se tiene conocimiento en base a la información primaria obtenida por los autores mediante entrevistas a comunidades de regantes, y a información secundaria procedente de fuentes públicas (Confederación Hidrográficas), privadas (asociaciones de regantes) y la bibliografía sobre el tema.

\section{Recursos y usos del agua en la cuenca del Segura}

Las estimaciones oficiales más recientes de recursos disponibles en la cuenca del Segura ascienden a una media de $1580 \mathrm{hm}^{3} /$ año, cifra que incluye las aguas superficiales y subterráneas, los recursos provenientes del Acueducto Tajo-Segura (ATS), la reutilización y la desalinización (CHS, 2014). Esta disponibilidad de recursos no basta para satisfacer la demanda bruta de agua de la cuenca que se estima en $1.841 \mathrm{hm}^{3} / a$ ño, de los que 1.546 $\mathrm{hm}^{3} /$ año corresponden a los usos agrarios, $236 \mathrm{hm}^{3} / a n ̃ o$ al abastecimiento urbano, 20

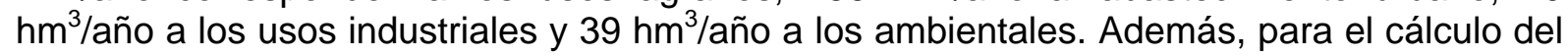
balance hídrico real de la cuenca no se consideran las extracciones de recursos subterráneos no renovables, por lo que los recursos renovables disponibles en la cuenca se estiman en $1.403 \mathrm{hm}^{3} /$ año, distribuidos en $1.113 \mathrm{hm}^{3} /$ año para las demandas agrarias, 236 $\mathrm{hm}^{3} /$ año para las urbanas, $15 \mathrm{hm}^{3} /$ año para las industriales y $39 \mathrm{hm}^{3} / a n ̃ o$ para las ambientales. Por lo tanto, considerando una demanda de $1.841 \mathrm{hm}^{3} / a n ̃ o$, se obtendría un déficit estructural de aproximadamente $438 \mathrm{hm} / a n ̃ o$. Este déficit se cubre mediante extracciones no renovables de recursos subterráneos (237 $\mathrm{hm}^{3} / \mathrm{año}$ ) y mediante una

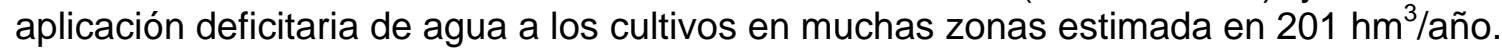

Como se ha mostrado, el principal usuario del agua en la cuenca del Segura es el regadío. La superficie regable de la cuenca asciende a 472.369 hectáreas, de las que 261.009 
hectáreas constituyen la superficie neta efectivamente regada (CHS, 2014). La superficie de regadío se ha multiplicado por dos desde los años 80 , lo que ha exacerbado la situación de escasez estructural de la cuenca y ha generado un grave problema de sobreexplotación en muchos acuíferos. Las zonas regables que se nutren del ATS, que incluyen algunas situadas en otras cuencas, comprenden un total de 152.488 hectáreas (CHS, 2015). La agricultura de regadío tiene gran importancia en la economía de la cuenca, tanto en términos del valor de la producción como del empleo y la actividad exportadora. MaestreValero et al. (2013) estiman que el valor medio de la producción agraria en el regadío de la cuenca del Segura asciende a 2.000 millones de euros anuales, lo que supone una rentabilidad para los agricultores en términos de margen neto de 865 millones de euros/año y genera un empleo anual equivalente a 58.500 Unidades de Trabajo Anual (UTA).

El valor de uso agrario del agua está entre los más altos del país. El valor marginal del agua en la cuenca es $0,52 € / \mathrm{m}^{3}$ y el valor medio $0,81 € / \mathrm{m}^{3}$, ambos medidos en términos de margen neto de explotación (Calatrava y Martínez-Granados, 2012). Sin embargo, estos valores presentan notables diferencias entre zonas, oscilando el valor marginal entre 0,13 y $0,97 € / \mathrm{m}^{3}$ y el valor medio entre 0,46 y $1,64 € / \mathrm{m}^{3}$. Los mayores valores corresponden a las zonas costeras de la cuenca y a las zonas regables que reciben agua del trasvase TajoSegura, debido a que en ellas se concentra la mayor parte de la horticultura intensiva y los invernaderos de la cuenca, además de tratarse generalmente de los regadíos más modernos y menos dotados en términos de disponibilidad de agua por hectárea.

En cuanto a las tarifas del agua, la tarifa media pagada en baja es casi $0,17 € / \mathrm{m}^{3}$ (CHS, 2014), muy por encima de la media de España (Calatrava et al., 2015). Por orígenes, las tarifas medias oscilan entre los $0,035 € / \mathrm{m}^{3}$ de los recursos superficiales y depurados, los $0,20 € / \mathrm{m}^{3}$ de los subterráneos y los $0,40 € / \mathrm{m}^{3}$ de los desalinizados. Estos valores medios son bastante homogéneos entre las diferentes áreas de la cuenca, con la excepción de las aguas subterráneas, cuyos costes de extracción varían entre $0,10-0,47 € / \mathrm{m}^{3}$ (CHS, 2014). A estas tarifas hay que añadir los costes de distribución del agua que los regantes pagan en forma de derramas a las comunidades de regantes, y que oscilan entre 0,04 y $0,075 € / \mathrm{m}^{3}$, con un valor medio de $0,06 € / \mathrm{m}^{3}$, según nuestras propias estimaciones en base a datos obtenidos de diversas comunidades de regantes. En el caso del abastecimiento urbano, el precio medio del agua potable suministrada a los municipios por la Mancomunidad de Canales del Taibilla (MCT), es de $0,6433 € / \mathrm{m}^{3}$.

\section{Contratos de cesión entre usuarios de la cuenca del Segura}

En la Figura 1 se muestran los volúmenes intercambiados y las compensaciones acordadas en los contratos de cesión autorizados en la cuenca entre 1999 y 2013, el período para el que se dispone de datos totales. El volumen medio anual es de casi $4 \mathrm{hm}^{3} / a n ̃ o$, lo que supone menos del $0,5 \%$ del agua utilizada en el regadío de la cuenca del Segura. Sin embargo, este valor medio es poco representativo, ya que solo se ha superado en tres de los trece años del período considerado, siendo el volumen intercambiado inferior a $2 \mathrm{hm}^{3}$ en la mitad de los años. Con algunas excepciones, el volumen medio anual intercambiado en cada contrato ha sido inferior a $0,4 \mathrm{hm}^{3} \mathrm{y}$, en muchos años, a $0,2 \mathrm{hm}^{3}$, si bien en el último lustro se han producido algunas operaciones superiores a los $4 \mathrm{hm}^{3}$.

En cuanto a las compensaciones acordadas, en primer lugar, la Figura 1 muestra un elevado grado de dispersión de los precios, lo que es de esperar dado el reducido nivel de concurrencia de este mercado. En segundo lugar, aunque las oscilaciones de los precios, tanto máximos como mínimos, son notables, se observan precios ligeramente superiores en los años de sequía. Finalmente, se observan menores precios en los primeros años del período, muy especialmente en los precios mínimos. Aclarar que todos los precios de intercambio citados en este trabajo son en origen y no incluyen ni el IVA ni los costes de transporte y distribución a los usuarios finales, salvo que se indique lo contrario. 


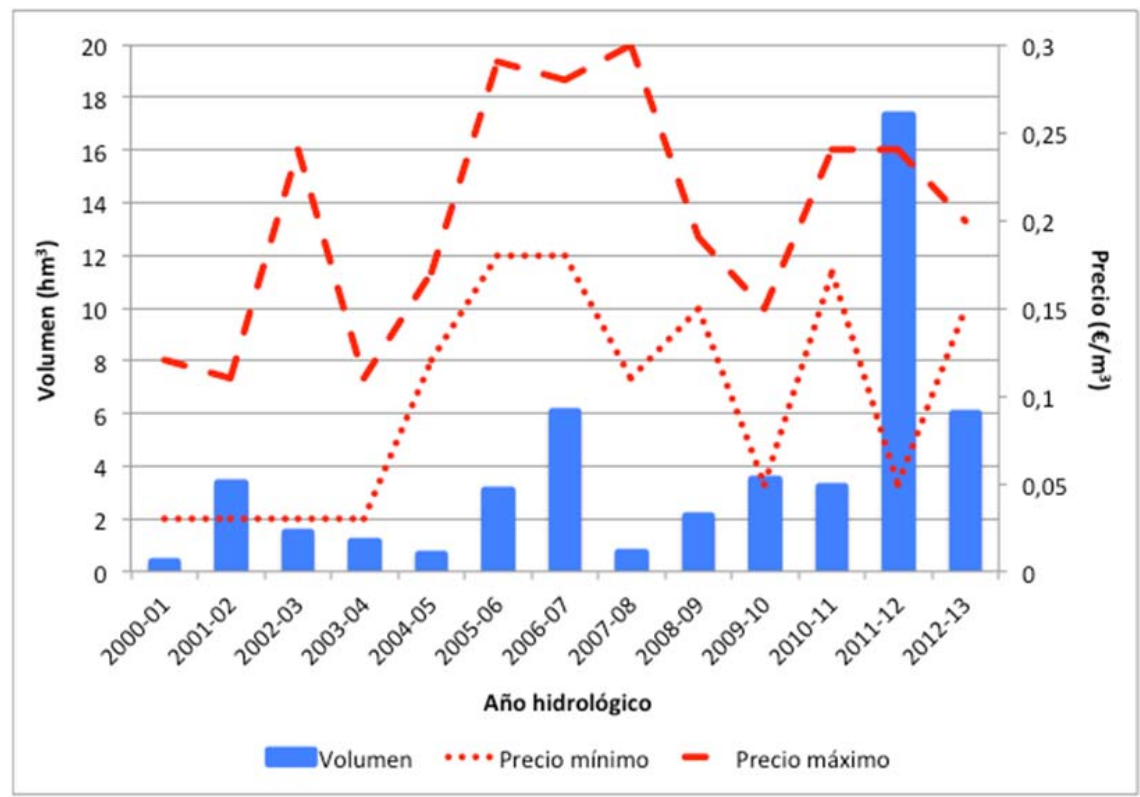

Figura 1. Volúmenes y precios en contratos de cesión en la cuenca del Segura (200013) Elaboración propia a partir de información proporcionada por la CHS.

De manera simplificada, las principales zonas de origen del agua intercambiada en contratos de cesión se localizan en la cabecera de la cuenca y a lo largo de la Vega del Río Segura. Las principales zonas de destino de los volúmenes adquiridos se localizan en el suroeste de la cuenca, más concretamente en las zonas del Alto Guadalentín, Águilas, Mazarrón y Bajo Almanzora, así como en las zonas regables del Acueducto Tajo-Segura. Esto no es óbice para que se hayan producido intercambios de ámbito espacial más restringido en otras zonas de la cuenca, aunque generalmente de menor volumen. El inusualmente elevado volumen de intercambios del año hidrológico 2011-12 se debe a la excepcional situación de abundancia de recursos superficiales propios en la cuenca en 2011 y 2012, lo que permitió una serie de operaciones de cierta importancia por parte de cedentes con exceso puntual de recursos y con destino principal en comunidades de regantes del suroeste de la cuenca.

Aunque no disponemos de información del volumen total intercambiado en los dos últimos años hidrológicos, la información disponible sobre algunos contratos recientes y de cierta relevancia, indica que los volúmenes intercambiados anualmente han sido superiores a la media de 2000-2013 y sugiere una tendencia creciente de los mismos, así como menores precios. Por ejemplo, en 2013-14 el Sindicato Central de Regantes del Acueducto TajoSegura (SCRATS) adquirió $1,2 \mathrm{hm}^{3}$ a la C.R. de Campotéjar y $4 \mathrm{hm}^{3}$ a la C.R. Riegos de Levante Margen Izquierda, ambas en la Vega del Segura. Por su parte, y también en 201314, las CC.RR. de Águilas y Mazarrón adquirieron $5 \mathrm{hm}^{3}$ de la Junta de Hacendados de la Huerta de Murcia, operación que se repitió en 2014-15. Este último acuerdo había sido denegado previamente por la imposibilidad física de medir los volúmenes consumidos por los cedentes, por lo que requirió de la instalación de un sistema de contadores volumétricos. El mismo problema ha frustrado otros acuerdos con el regadío tradicional, caso del alcanzado en 2015 por el SCRATS con el Juzgado Privativo de Aguas de Orihuela.

Algunos de estos acuerdos no han estado exentos de polémica. Las cesiones de agua de la Junta de Hacendados de la Huerta de Murcia a las CC.RR. de Águilas y Mazarrón en 2014 y 2015 fueron recurridas por la Asociación para la Conservación de la Huerta de Murcia (HUERMUR) ante la Confederación Hidrográfica del Segura (CHS), quien desestimó dicho recurso. Mayor oposición se ha encontrado el SCRATS en 2015 en su intento de adquirir agua a varias CC.RR. del regadío tradicional de la Vega Baja del Segura, que viene 
manteniendo a lo largo de los años una postura de oposición a las cesiones de agua desde la Vega del Segura a otras zonas de la cuenca. Más recientemente, ya en 2016, la propuesta de ceder $30 \mathrm{hm}^{3}$ del regadío tradicional de la cuenca al SCRATS ha desatado una tormenta política que ha requerido la intervención directa del MAGRAMA.

\section{Contratos de cesión con usuarios de la cuenca del Tajo}

La lógica de funcionamiento de los mercados de agua, que supone la transferencia de los recursos de usos de menor valor a otros de mayor valor, hace pensar en los usuarios urbanos como los primeros y principales compradores, como ocurre en muchas zonas del suroeste de los EE.UU. Sin embargo, la prioridad que los abastecimientos tienen en España sobre otros usos del agua, ha limitado en la práctica el recurso a los contratos de cesión. Aunque hay experiencias de acuerdos puntuales en las cuencas del Tajo, Segura y Júcar, algunos anteriores a la Ley de Aguas de 1999, la mayoría de los intercambios formales de agua en España han tenido lugar entre usuarios agrarios (Palomo-Hierro et al., 2015).

En el caso del Segura, la Mancomunidad de Canales del Taibilla (MCT) firmó durante la sequía de 2005-2008 tres convenios con la C.R. del Canal de Las Aves, situada en Aranjuez (Madrid), para la cesión de un máximo anual de $40 \mathrm{hm}^{3}$ (Tabla 1). Sin embargo, el objetivo real de estos convenios era crear una reserva estratégica en la cabecera del Tajo que asegurase el abastecimiento impidiendo que los niveles de los embalses de Entrepeñas y Buendía, de los que se nutre el ATS, cayesen por debajo del umbral crítico que imposibilita por ley el trasvase de recursos al Segura (Calatrava y Gómez-Ramos, 2009). En total, la MCT pagó 30,33 millones de euros por un volumen adquirido de $108,5 \mathrm{hm}^{3}$, equivalente a un precio medio de $0,2795 € / \mathrm{m}^{3}$, si bien en la práctica solo se trasvasaron $47,78 \mathrm{hm}^{3}\left(2,34 \mathrm{hm}^{3}\right.$

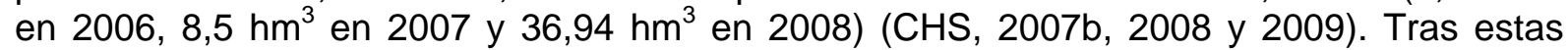
operaciones, y alguna compra puntual de pequeños volúmenes a usuarios de la propia cuenca durante la sequía de 2005-08, la entrada en funcionamiento de las desalinizadoras de San Pedro del Pinatar y Alicante, así como un mayor recurso a pozos de sequía de la $\mathrm{CHS}$, ha permitido que la MCT no haya vuelto a recurrir a las compras de agua.

Tabla 1. Contratos de cesión inter-cuencas entre usuarios del Segura y del Tajo

\begin{tabular}{cccccc}
\hline \multirow{2}{*}{ Comprador } & Cedente & $\begin{array}{c}\text { Año } \\
\text { hidrológico }\end{array}$ & $\begin{array}{c}\text { Volumen en } \\
\text { origen }\left(\mathbf{h m}^{3}\right)\end{array}$ & $\begin{array}{c}\text { Precio } \\
\left(\boldsymbol{\epsilon} / \mathbf{m}^{3}\right)\end{array}$ & $\begin{array}{c}\text { Compensación } \\
\text { (euros) }\end{array}$ \\
\hline \multirow{2}{*}{ MCT } & C.R. Canal de & $2005-2006$ & 35,52 & 0,2885 & 10.247 .520 \\
& las Aves & $2006-2007$ & 36,03 & 0,2364 & 8.517 .492 \\
& $2007-2008$ & 36,94 & 0,3130 & 11.562 .220 \\
\hline \multirow{3}{*}{ SCRATS } & $2005-2006$ & 31,05 & 0,1856 & 5.761 .700 \\
& & $2006-2007$ & 31,05 & 0,1881 & 5.839 .480 \\
& C.R. Canal de & $2007-2008$ & 31,05 & 0,1920 & 5.962 .097 \\
& Estremera & $2008-2009$ & 31,05 & 0,1920 & 5.962 .097 \\
& & $2013-2014$ & 5,560 & 0,06 & 333.600 \\
& & $2014-2015$ & 7,7 & 0,06 & 462.000 \\
\cline { 2 - 6 } & \multirow{2}{*}{ C.R. La Poveda } & $2013-2014$ & 1,4 & 0,06 & 84.000 \\
& $2014-2015$ & 1,4 & 0,06 & 84.000 \\
\hline
\end{tabular}

Fuente: Elaboración propia a partir de Claver (2013), SCRATS (2009 y 2015), las memorias anuales de la MCT (2007, 2008 y 2009), y de datos adicionales proporcionados por los compradores. Precios en origen sin incluir ni el I.V.A. ni la tarifa del trasvase Tajo-Segura correspondiente. 
Por su parte, el SCRATS estableció cuatro convenios sucesivos entre 2006 y 2009 con la C.R. del Canal de Estremera para la cesión anual de $31,05 \mathrm{hm}^{3}$ medidos en origen (Claver, 2013). Dicha comunidad de regantes renunciaba a utilizar la totalidad de su concesión en el período establecido en cada contrato de cesión, durante los cuales llevó a cabo la mejora y modernización de toda la zona regable. La compensación económica acordada el primer año fue de $0,1855 € / \mathrm{m}^{3}$, valor que fue incrementándose en una cuantía igual al $50 \%$ del valor del IPC de cada año, con excepción del cuarto contrato (Tabla 1). En la práctica, estos precios unitarios son mayores, ya que hay que considerar un $10 \%$ por pérdidas en el transporte a través del ATS y añadir el componente a) de la tarifa del trasvase $(0,013856$ $\left.€ / \mathrm{m}^{3}\right)$ y las cuotas del SCRATS $\left(0,002404 € / \mathrm{m}^{3}\right)$, lo que incrementa los precios medidos en las tomas de las CC.RR del ATS por encima de los $0,22 € / \mathrm{m}^{3}$ (Claver, 2013). A estos valores habría que añadir las derramas a pagar por los regantes a cada comunidad de regantes.

Los acuerdos entre el SCRATS y la C.R. del Canal de Estremera permitieron mitigar la escasez de agua en las zonas regables del ATS y fueron beneficiosos para ambas partes. Calatrava y Gómez-Limón (2016) valoran las ganancias brutas de este acuerdo plurianual en 89,9 millones de euros para los usuarios del ATS y 17,8 millones de euros para los de la C.R. del Canal de Estremera. Además, esta última utilizó los pagos por la cesión de derechos para financiar la modernización de sus infraestructuras. Sin embargo, estos acuerdos también dieron lugar a protestas por parte de usuarios aguas abajo en el Tajo (Garrido et al., 2013b). Cuando la situación de sequía se ha repetido en 2014, se ha reactivado este acuerdo, aunque con diferentes condiciones.

Los dos casos de cesiones inter-cuencas de 2005-2009 recibieron el apoyo explícito del Ministerio de Medio Ambiente, que permitió dotaciones de agua intercambiadas muy elevadas (Calatrava y Gómez-Ramos, 2009), y se beneficiaron de exenciones en la tarifa del Acueducto Tajo-Segura, equivalentes a $0,085774 € / \mathrm{m}^{3}$, justificados por el Gobierno de España en base a la situación de extrema sequía existente (Garrido et al., 2013b). En concreto, quedaron exentos del pago de las cuotas correspondientes a los apartados b) y c) de la tarifa del trasvase, por lo que solo pagaron el apartado a) de la tarifa, que corresponde a la amortización del coste de las obras y cuyo importe va destinado a las CC.AA. por las que discurre el río Tajo. De hecho, puesto que estas exenciones se aplicaron tanto a los volúmenes procedentes de los contratos de cesión como a los volúmenes ordinarios trasvasados desde el Tajo, su cuantía económica superó el coste de adquisición por parte del SCRATS de los volúmenes de la C.R. del Canal de Estremera (Hernández-Mora y Del Moral, 2015). En el caso de los acuerdos entre la MCT y la C.R. del Canal de las Aves, el coste total de las compras $(30,687$ millones de euros) se compensó con la exención del peaje de los volúmenes ordinarios del trasvase durante la sequía de 2005-2008 (27,977 millones de euros) y de los propios volúmenes adquiridos (4,098 millones de euros).

Frente al apoyo del Gobierno Central, estos acuerdos de cesión recibieron fuerte oposición por parte del Gobierno de Castilla-La Mancha que recurrió sucesivamente en los tribunales los contratos de cesión de 2007, 2008 y 2009, habiendo sido desestimado hasta la fecha el primero de estos recursos. Es evidente que esta conflictividad supone un coste político de estos intercambios que no debe ser desdeñado. Menos clara es la evidencia sobre posibles efectos ambientales de los contratos inter-cuencas de 2005-2009, ya que la Ley 21/2013 solo exige una evaluación de impacto ambiental previa para trasvases superiores a $100 \mathrm{hm}^{3}$ pero no para cesiones temporales de derechos (Claver, 2013). Mientras que Yagüe (2008) y Claver (2013) mantienen que las cesiones entre el Tajo y el Segura no causaron problemas medioambientales, ya que se mantuvo el caudal ambiental mínimo en el tramo del río Tajo afectado, Hernández-Mora (2013) mantiene que los impactos fueron severos, ya que los caudales en Aranjuez cayeron por debajo de los $6 \mathrm{~m}^{3} / \mathrm{s}$ en diversas ocasiones.

Posteriormente, el mismo SCRATS firmó un acuerdo en 2011 con la C.R. de Illana-Leganiel en el Alto Tajo para la cesión total o parcial de su dotación durante 10 años (SCRATS, 
2012). La compensación económica pactada en el acuerdo tiene dos componentes: un pago anual fijo equivalente al canon de regulación de dicha comunidad de regantes (entre 13.000 $€$ y $17.000 €$ según el año) y un pago variable de $0,06 € / \mathrm{m}^{3}$ por el agua cedida en el caso de ser autorizada la cesión. Este contrato presenta notables semejanzas con un contrato de opción de suministro de agua, ya que la C.R. de Illana-Leganiel se compromete a ceder el volumen pactado cuando se den determinadas condiciones: una situación de sequía, que se active legalmente la posibilidad de celebrar cesiones inter-cuenca y que el SCRATS así lo solicite. En la práctica, cuando se han dado estas circunstancias en 2014 y 2015, la Dirección General del Agua ha denegado la solicitud de autorización de este contrato por considerar que la comunidad de regantes cedente no ha hecho uso de sus recursos en los últimos años, requisito previo para autorizar cualquier contrato de cesión (SCRATS, 2015).

Más recientemente, en 2014 y 2015 se han producido otros dos acuerdos entre el SCRATS y dos comunidades de regantes del Alto Tajo (Tabla 1). En primer lugar, se han firmado dos nuevos contratos con la C.R. del Canal de Estremera por un volumen de $9 \mathrm{hm}^{3}$ anuales, si bien la Dirección General del Agua solo ha autorizado la cesión de 5,56 hm ${ }^{3}$ en 2013-14 y $7,7 \mathrm{hm}^{3}$ en 2014-15. Estas denegaciones parciales se deben al hecho de que el consumo real acreditado por esta comunidad de regantes en 2013 y 2014 respectivamente es inferior al volumen solicitado. En los mismos años, el SCRATS firmó otros dos contratos de cesión con la C.R. de La Poveda (Madrid) que fueron autorizados por un volumen de 1,4 $\mathrm{hm}^{3} /$ año. Es de importancia recalcar que estos nuevos acuerdos no se han beneficiado de la exención de la tarifa del ATS, como en el caso de la anterior sequía, por lo que a los precios indicados en la Tabla 1 hay que añadir, además del IVA, 0,0984 €/m³ en concepto de dicha tarifa.

\section{Ofertas públicas de adquisición de derechos}

Aunque el Centro de Intercambio de Derechos (CID) de agua del Segura se creó en 2004, no fue hasta 2007 cuando la CHS lanzó una primera OPAD con el objetivo de crear una reserva estratégica para garantizar los caudales ambientales en los ríos Segura y Mundo, en la cabecera de la cuenca (CHS, 2007a). El presupuesto de la OPAD era de 700.000 euros y la compensación máxima a pagar por la cesión de derechos era $0,18 € / \mathrm{m}^{3}$, siendo los costes del transporte del agua asumidos por la CHS. Finalmente, se aceptaron 41 ofertas de pequeños agricultores que sumaban un total de 371,5 hectáreas. El volumen adquirido fue $2,93 \mathrm{hm}^{3}$, a un precio medio de $0,168 € / \mathrm{m}^{3}$ y con un coste de 495.000 euros, y se destinó en si totalidad a usos ambientales (Garrido et al., 2013b). La OPAD se repitió en 2008 con idénticas condiciones y similares resultados (Calatrava y Gómez-Ramos, 2009).

Al igual que en las cuencas del Guadiana y del Júcar, el éxito de las OPAD del Segura fue moderado, ya que no se agotó el presupuesto inicial por falta de oferentes que cumplieran con los requisitos exigidos (Rey et al., 2014). Para Yagüe (2008), el precio máximo establecido era atractivo para los regantes, pero pudo más la "desconfianza que generan estos nuevos instrumentos entre los regantes y la cultura existente de mantener a ultranza los derechos de agua". En la práctica, muchos de los arroceros que participaron lo hicieron porque la disponibilidad de agua no les permitía completar sus ciclos de cultivo.

\section{Discusión}

Las experiencias de mercados de agua en la cuenca del Segura abarcan desde intercambios informales a nivel local, contratos de cesión entre usuarios de la Demarcación (amparados por la Ley 46/1999) o entre usuarios de diferentes cuencas (amparados por el Real Decreto 15/2005 y posteriores) y ofertas públicas de adquisición de derechos. Dejando a un lado los mercados informales por su casi imposible cuantificación, podemos afirmar que la actividad de los mercados formales de agua en el Segura ha sido menor de lo que se pudiera haber previsto dadas las condiciones hidrológicas y las características de la economía del agua en la cuenca, y que se ha limitado casi totalmente al ámbito agrario. 
Frente a los acuerdos intra-cuenca $\left(49,12 \mathrm{hm}^{3}\right)$, la mayoría de los volúmenes adquiridos en 2000-2013 proceden de acuerdos con usuarios del Tajo $\left(232,69 \mathrm{hm}^{3}\right)$.

Al igual que lo sucedido a nivel nacional, la actividad e impacto de los intercambios de agua en la cuenca del Segura durante los últimos tres lustros ha sido limitada y desigual. De manera resumida, podemos decir que en los últimos años los contratos de cesión se reducen a una o dos grandes operaciones puntuales (3-6 $\left.\mathrm{hm}^{3}\right)$ cada año, casi siempre con las mismas zonas compradoras, y un número algo mayor, en torno a la decena, de pequeñas operaciones entre usuarios privados agrarios o pequeñas comunidades de regantes por volúmenes generalmente inferiores a $0,25 \mathrm{hm}^{3}$. Sin embargo, mientras que a nivel nacional la actividad de los mercados de agua ha estado concentrada en intercambios inter-cuencas en épocas de sequía, en el caso del Segura ésta se mantiene incluso en años de mayor disponibilidad de recursos, debido al desigual reparto de los recursos en la cuenca. El potencial de intercambio de agua en la cuenca no se limita por tanto a situaciones de escasez puntual, como tampoco es necesaria una situación coyuntural de sequía para que exista demanda de intercambios con los usuarios del Tajo, sino que dicha demanda existe también en años normales.

Otro aspecto de relevancia son las compensaciones acordadas en los intercambios. Se observa un notable grado de dispersión de los precios con mínimos en torno a $0,06 € / \mathrm{m}^{3}$ y $0,24 € / \mathrm{m}^{3}$ (incluso mayores en el caso de compras realizadas para usos urbanos), si bien no hay que olvidar que a estos valores hay que añadir los costes de transporte, elevación y distribución del agua que en algunos casos extremos han llegado a duplicar el coste final para el agricultor. Se observa también una evolución ligeramente decreciente de los precios hasta valores medios en la actualidad entorno a $0,15 € / \mathrm{m}^{3}$ (netos en origen). Sorprende también que los precios no guarden relación directa con el valor medio del recurso en las zonas de origen y/o destino, sino que parecen depender más de la capacidad de negociación y las circunstancias puntuales de escasez para ambas partes.

El elevado grado de dispersión de los precios, junto con el relativamente escaso número de transacciones de agua, apunta a la existencia de un mercado "estrecho" (thin market), típico de situaciones en las que el número de potenciales participantes en el mercado es reducido debido al limitado ámbito espacial del mercado, por ejemplo, porque existan restricciones físicas o legales al intercambio (Tisdell, 2011), lo que genera falta de competencia y dispersión en los precios (que dejan de ser un buen indicador de escasez).

En el caso de las OPAD, la experiencia del Segura es similar a la de otras cuencas, ya que no se agotó el presupuesto de adquisición. Los valores del agua en las zonas cedentes, en la parte castellano-manchega de la cuenca, sugieren que más que un problema de precios de oferta bajos, han primado otros comportamientos de tipo estratégico y/o colusivo, o que han existido presiones externas sobre los regantes para no participar en las OPAD (Calatrava y Gómez-Ramos, 2009).

Obviamente, un aspecto positivo es que los recursos se han reasignado a los usos de mayor valor. En el caso de los contratos inter-cuencas, los resultados obtenidos por Calatrava y Gómez-Limón (2016) sugieren que las ganancias de bienestar han sido cuantiosas y que pueden superar los posibles impactos ambientales negativos, a falta de una cuantificación de estos últimos. En este sentido, y a nivel de la cuenca, los volúmenes intercambiados y la denegación de determinadas solicitudes permite suponer que los posibles impactos ambientales han sido insignificantes, habiéndose producido incluso beneficios ambientales como consecuencia de las OPAD que, como en el resto de España, y de manera similar a los bancos públicos del agua de California o Australia (Wheeler at al., 2013), se han utilizado solo para la consecución de objetivos medioambientales (Garrido et al., 2013a). En el caso de los contratos de cesión inter-cuencas, la lógica sugiere que los impactos ambientales han debido ser mayores, si bien las opiniones al respecto son contradictorias. En cualquier caso, 
una de las debilidades más claras del marco legal de los mercados de agua en España es la no consideración de los efectos ambientales, positivos o negativos (Calatrava y GómezRamos, 2009), más que el impacto ambiental resultante de un intercambio concreto y puntual que seguramente no sea irreversible (Garrido et al., 2013c).

Pese al importante papel de la Administración como promotor de los intercambios intercuencas durante la sequía (Garrido et al., 2013a) y a las sucesivas reformas legales para ampliar el alcance de los intercambios (Hernández-Mora y Del Moral, 2015), no está clara la apuesta de la Administración por aprovechar las posibilidades de los centros de intercambios para facilitar los intercambios, agrupando la oferta, reduciendo los costes de transacción y promoviendo una mayor participación de los usuarios.

Las experiencias de mercados e agua en el Segura, con sus claroscuros, han sido positivas, ya que han permitido evitar importantes perjuicios económicos en zonas y momentos puntuales, facilitando incluso la consecución de objetivos medioambientales a través de las OPAD. Sin embargo, pese a sus beneficios, los mercados de agua no son la solución al problema de la escasez estructural en la cuenca, sino un instrumento más de los que pueden utilizarse, y, en ningún caso, pueden sustituir la acción de la Administración. Es más, lo limitado de su alcance en la práctica plantea dudas sobre su potencial real en épocas de sequía, en las que otras actuaciones, como la movilización de recursos subterráneos estratégicos y el recurso a la desalinización, han suministrado mayores volúmenes a los regantes.

\section{Agradecimientos}

La realización de este trabajo ha sido posible gracias a la financiación del Ministerio de Economía y Competitividad y el Fondo Europeo de Desarrollo Regional (FEDER) mediante el proyecto MERCAGUA (AGL2013-48080-C2-2-R) y del Programa de Apoyo a la Investigación de la Fundación Séneca-Agencia de Ciencia y Tecnología de la Región de Murcia mediante el proyecto 19280/PI/14.

\section{Referencias}

Calatrava, J., García-Valiñas, M.A., Garrido, A. y González-Gómez, F. (2015). Water pricing in Spain: Following the footsteps of somber climate change projections; en Dinar, A., Pochat, V. y Albiac, J., eds.: Water pricing experiences and innovations. Springer, Cham, Switzerland; pp. 313-340.

Calatrava, J. y Gómez-Ramos, A. (2009). El papel de los mercados de agua como instrumento de asignación de recursos hídricos en el regadío español; en Gómez-Limón, J. A., Calatrava, J., Garrido, A., Sáez, F. J. y Xabadia, À., eds.: La economía del agua de riego en España. Fundación Cajamar, Almería. pp 295-319.

Calatrava, J. y Gómez-Limón, J. A. (2016). Mercados de agua y eficiencia económica; en Gómez-Limón, J. A. y Calatrava, J., eds.: Los mercados de agua en España: Presente y perspectivas. Cajamar Caja Rural, Almería.

Calatrava, J. y Martínez-Granados, D. (2012). El valor de uso del agua en el regadío de la cuenca del Segura y en las zonas regables del trasvase Tajo-Segura. Economía Agraria y Recursos Naturales 12(1): 5-32.

CHS (2007a). Pliego de cláusulas administrativas particulares y prescripciones técnicas particulares que regirán en la oferta publica de la Confederación Hidrográfica del Segura para la adquisición de derechos de agua con destino a la cuenca del Segura por razones de garantía de los caudales ambientales y de abastecimiento de poblaciones. Confederación Hidrográfica del Segura, Murcia.

CHS (2007b). Memoria 2006. Confederación Hidrográfica del Segura, Murcia.

CHS (2008). Memoria 2007. Confederación Hidrográfica del Segura, Murcia. 
CHS (2009). Memoria 2008. Confederación Hidrográfica del Segura, Murcia.

CHS (2014). Borrador del Plan Hidrológico de la Cuenca del Segura 2015-2021. Confederación Hidrográfica de la Cuenca del Segura, Murcia.

CHS (2015). Estudio General sobre la Demarcación Hidrográfica del Segura. Confederación Hidrográfica del Segura, Murcia.

Claver, J. M. (2013). La experiencia de un contrato de cesión de derechos de aguas intercuencas (el derecho al aprovechamiento de las aguas trasvasadas); en Embid, A., ed.: Usos del agua (concesiones, autorizaciones y mercados de agua). AranzadiThomson Reuters, Cizur Menor (España).

Garrido, A., Maestu, J., Gomez-Ramos, A., Estrela, T., Yague, J., Segura, R., Calatrava, J., Arrojo, P. y Cubillo, F. (2013a). Voluntary water trading in Spain: A mixed approach of public and private initiatives; en Maestu, J., ed.: Water trading and global water scarcity: International experiences. Routledge, Abingdon, U.K. pp. 180-193.

Garrido, A., Rey, D. y Calatrava, J. (2013b). Water trading in Spain; en De Stefano, L. y Llamas, M. R., eds.; Water, agriculture and the environment in Spain: Can we square the circle? Botín Foundation, CRC Press, Boca Raton (CA); pp. 205-216.

Garrido A., Calatrava J. y Rey D. (2013c). La flexibilización del régimen de concesiones y el mercado de aguas en los usos de regadío; en Embid, A., ed.: Usos del agua (concesiones, autorizaciones y mercados de agua). Aranzadi-Thomson Reuters, Cizur Menor (España); pp. 177-197.

Hernández-Mora, N. (2013). El Tajo: Historia de un río ignorado. Fundación Nueva Cultura del Agua, Zaragoza.

Hernández-Mora, N. y Del Moral, L. (2015). Developing markets for water reallocation: Revisiting the experience of Spanish water mercantilización. Geoforum 62: 143-155.

Maestre-Valero, J. F., Martínez-Granados, D., Martínez-Alvarez, V. y Calatrava, J. (2013). Socio-economic impact of evaporation losses from reservoirs under past, current and future water availability scenarios in the semi-arid Segura basin. Water Resources Management 27(5): 1411-1426.

MCT (2007). La gestión del servicio 2006. Mancomunidad de los Canales del Taibilla, Ministerio de Medio Ambiente, Cartagena, España.

MCT (2008). La gestión del servicio 2007. Mancomunidad de los Canales del Taibilla, Ministerio de Medio Ambiente, Cartagena, España.

MCT (2009). La gestión del servicio 2008. Mancomunidad de los Canales del Taibilla, Ministerio de Medio Ambiente, Cartagena, España.

Palomo-Hierro, S., Gómez-Limón, J.A. y Riesgo, L. (2015). Water Markets in Spain: Performance and Challenges. Water 7: 652-678.

Rey D., Garrido A. y Calatrava J. (2014). Water markets in Spain: Meeting twenty-first century challenges with twentieth century regulations; en Easter, K. W. y Huang, Q., eds.; Water markets for the 21st. century: What have we learned? Springer, Dordrecht, The Netherlands; pp. 127-147.

SCRATS (2009): Memoria 2008. Sindicato Central de Regantes del Acueducto Tajo-Segura, Murcia.

SCRATS (2012): Memoria 2011. Sindicato Central de Regantes del Acueducto Tajo-Segura, Murcia.

SCRATS (2015): Memoria 2014. Sindicato Central de Regantes del Acueducto Tajo-Segura, Murcia.

Tisdell, J. G. (2011). Water markets in Australia: An experimental analysis of alternative market mechanisms. Australian Journal of Agricultural and Resource Economics 55(4): 500-517.

Wheeler, S., Garrick, D., Loch, A., y Bjornlund, H. (2013). Evaluating water market products to acquire water for the environment in Australia. Land Use Policy 30(1): 427-436.

Yagüe, J. (2008). Experiencia de los instrumentos de mercado en España; Thematic week seven: Economics and financing - The role of market instruments in integrated water management. Tribuna del Agua, Expo Agua 2008, Zaragoza. 